\title{
Research on construction of Web 3D-GIS based on Skyline
}

\author{
Tingting Wang ${ }^{* a}$, Zhiqiang Gao ${ }^{\text {b,c }}$, Jicai Ning ${ }^{\mathrm{b}}$ \\ ${ }^{a}$ Institute of Geographic Sciences and Natural Resources Research, Chinese Academy of Sciences, \\ Beijing, 100101,China; ' 'Yantai Institute of Coastal Zone Research, Chinese Academy of Sciences, \\ Yantai, 264003, China; ${ }^{\mathrm{c} U S D A}$ UV-B Monitoring and Research Program and Center of Remote \\ Sensing and Modeling for Agricultural Sustainability, Natural Resource Ecology Laboratory, \\ Colorado State University, Fort Collins, CO, USA
}

\begin{abstract}
This paper further studies the construction, publishing and display of three-dimensional (3D) scenes and their implementation based on Skyline family of software, combining remote sensing images and DEM data. Among them, the SketchUp software is used to build landscape models and the JavaScript programming language is adopted to achieve web browsing of 3D scenes. The study provides a useful exploration for the establishment of Web 3D-GIS combining Web GIS technology and 3D visualization technology.
\end{abstract}

Keywords: Web GIS, 3D GIS, Skyline.

\section{INTRODUCTION}

With the deepening of GIS applications and the increasing requirements for geographic information from various sectors, the traditional two-dimensional GIS has been unable to meet the needs of society. Because of a better description of the real three-dimensional objective world, 3D GIS has become an important research area of GIS.

In recent years, due to the rapid development of Internet technology, the conventional terminal/host-based and LAN-based GIS has been unable to satisfy the information need of the Internet era ${ }^{1}$. Web GIS combines the Internet technology with GIS and its distributed computing model facilitates information sharing and application integration ${ }^{2}$.

Web 3D-GIS uses virtual reality and visualization technology to construct a dynamically interactive virtual three-dimensional system within the network environment ${ }^{3}$. It combines the advantages of 3D GIS and Web GIS and achieves genuine and open geographic information sharing. Related researches apply it to park planning ${ }^{4}$, flood simulation $^{5}$, forest fire spread ${ }^{6}$, anti-terrorism security ${ }^{7}$, high-speed traffic ${ }^{8}$, campus view $^{9}$ and other fields.

This paper discusses the combination of 3D GIS and Web GIS and tries to create a Web three-dimensional visualization system based on Skyline series software. The study builds a three-dimensional scene of the study area and publishes it on web, thereby showing the three-dimensional terrain and landscape scene of the area, providing remote users' web browsing and sharing information. This paper is a simple attempt to build Web 3D-GIS and provides a reference for related research on Web GIS and 3D GIS.

\section{METHODOLOGY}

With the Skyline series software as platform, the study constructs a three-dimensional scene and releases the three-dimensional terrain of the study area, which is a watershed of Yantai-Weihai Region in Shandong Province, China. In addition, the scene is published with IIS server, and the web browsing of the scene is achieved with JavaScript scripting language, and ultimately a case of Web 3D-GIS is created. The Skyline series software is mainly composed of TerraBuilder, TerraExplorer Pro and TerraGate.

The construction of Web 3D-GIS includes data collection, data processing, scene creation and web publishing. The specific process is shown in Figure 1.

*Corresponding author. Email: wangtt_9955@126.com

Remote Sensing and Modeling of Ecosystems for Sustainability XI, edited by Wei Gao, Ni-Bin Chang, Jinnian Wang, Proc. of SPIE Vol. 9221, 92210I

(c) 2014 SPIE · CCC code: $0277-786 X / 14 / \$ 18 \cdot$ doi: $10.1117 / 12.2058581$ 


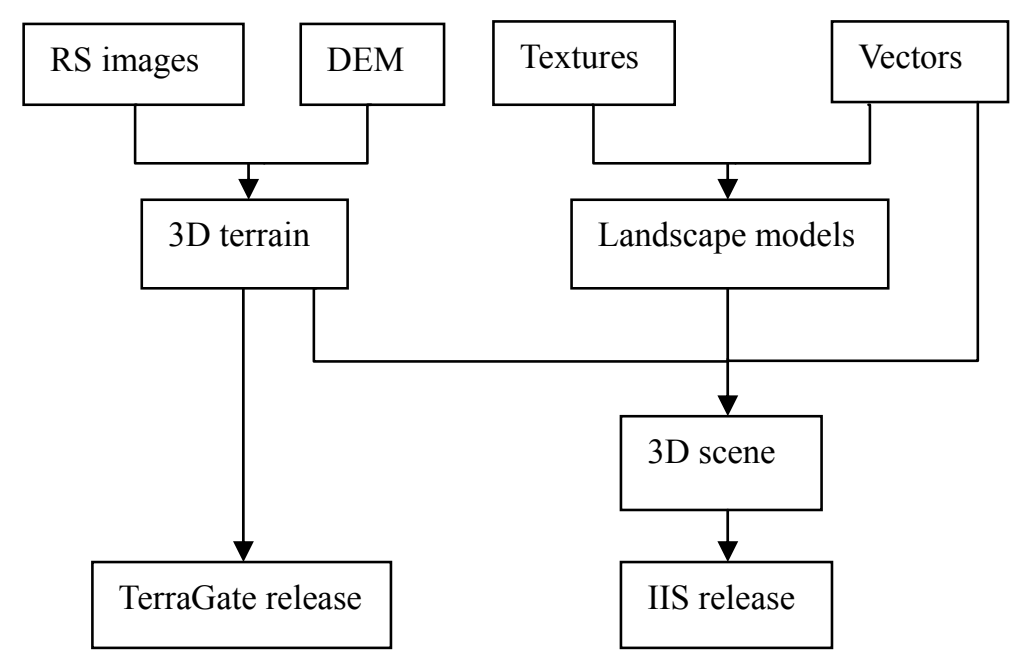

Figure 1. Construction procedure of Web 3D-GIS

\section{RESULTS AND DISCUSSION}

\subsection{Data and preprocessing}

The data needed for creation of Web 3D-GIS contains remote sensing images or aerial images, DEM data, two-dimensional vector data and landscape texture data. This study adopts high-resolution remote sensing images obtained from Google Earth, 30m resolution DEM data downloaded from the Chinese Academy of Sciences website, and vector data including layers of buildings and roads acquired by vectorizing the images.

The preprocessing of data includes georeferencing and color matching of remote sensing images, coordinate system conversion and format conversion of data.

\subsection{Construction of three-dimensional scene}

Three-dimensional scene consists of three-dimensional terrain, landscape models and two-dimensional vectors.

The creation of three-dimensional terrain is completed in TerraBuilder software, which includes overlaying remote sensing image and DEM data, cutting out the target area and generating pyramid data structures. Tools in TerraBuilder are used for editing including calibrating, removing background value, checking adjacent edges and color matching. When the image matches well with the DEM data, three-dimensional terrain is created in MPT format.

Landscape models refer to various 3D models in three-dimensional scene, the creation of which contains establishment of geometry models and texture mapping. This study adopts SketchUp software to build 3D models. The drawing tools of the software are employed to create geometry models, with vector layers to provide spatial positioning and texture mapping to improve the fidelity of models. Completed models are exported in 3DS format and then converted to X format using relevant plug-in since TerraExplorer software fails to support 3DS files.

TerraExplorer Pro software is used to build three-dimensional scene, which integrates three-dimensional terrain, landscape models, vector data and pyramid data structures built to optimize landscape models. After editing such as adding annotations, dynamic paths and messages the scene is exported in FLY format. The created scene is shown in Figure 2 .

\subsection{Web publishing and browsing}

TerraGate, a network data server, is utilized to publish terrain data which contains adding the MPT file directory to TerraGate and setting IP address, port number and other relevant settings. The client can obtain the published MPT file via TerraExplorer with a format as MPT file name@IP address: port number. TerraGate is unable to release scene files in FLY format. 
The scene data are published by IIS server with the FLY files placed in a virtual directory under IIS site and associated options set. The client can browse the released FLY files through TerraExplorer with an address as http://ip/virtual directory/FLY file name.

TerraExplorer Pro provides a set of ActiveX controls that allow the users embed three-dimensional windows, information trees and navigation maps in a custom interface as ActiveX objects. Adopting JavaScript scripting language, this paper adds three-dimensional window and information tree to a HTML page via object label to achieve web browsing of three-dimensional scene. Figure 3 shows the effect of viewing the scene on a page.

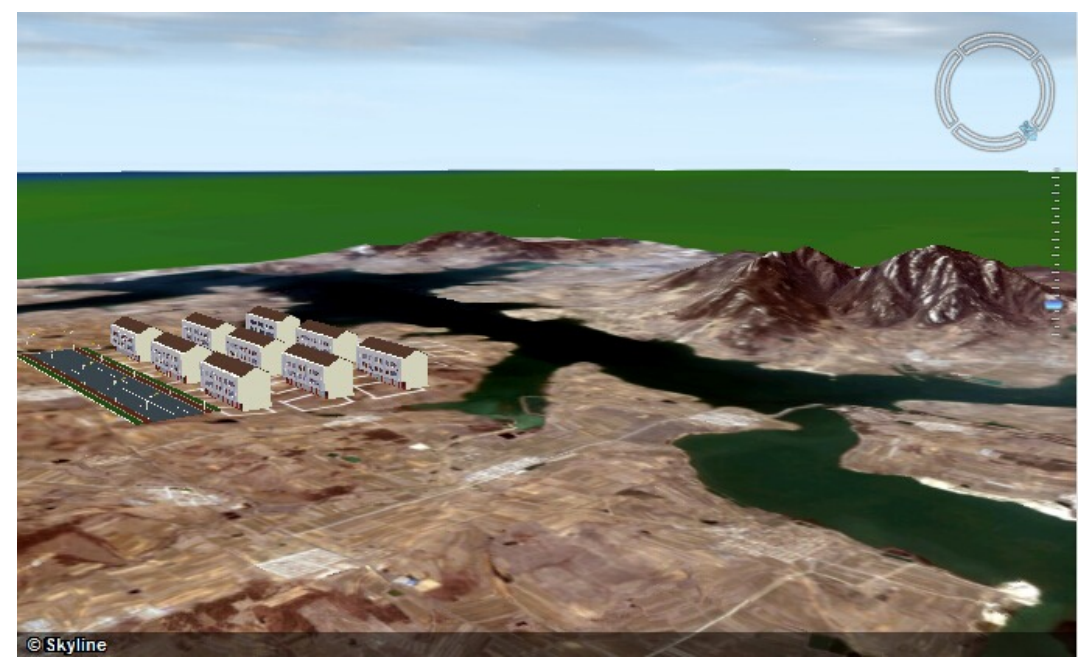

Figure 2. Three-dimensional scene of the study area

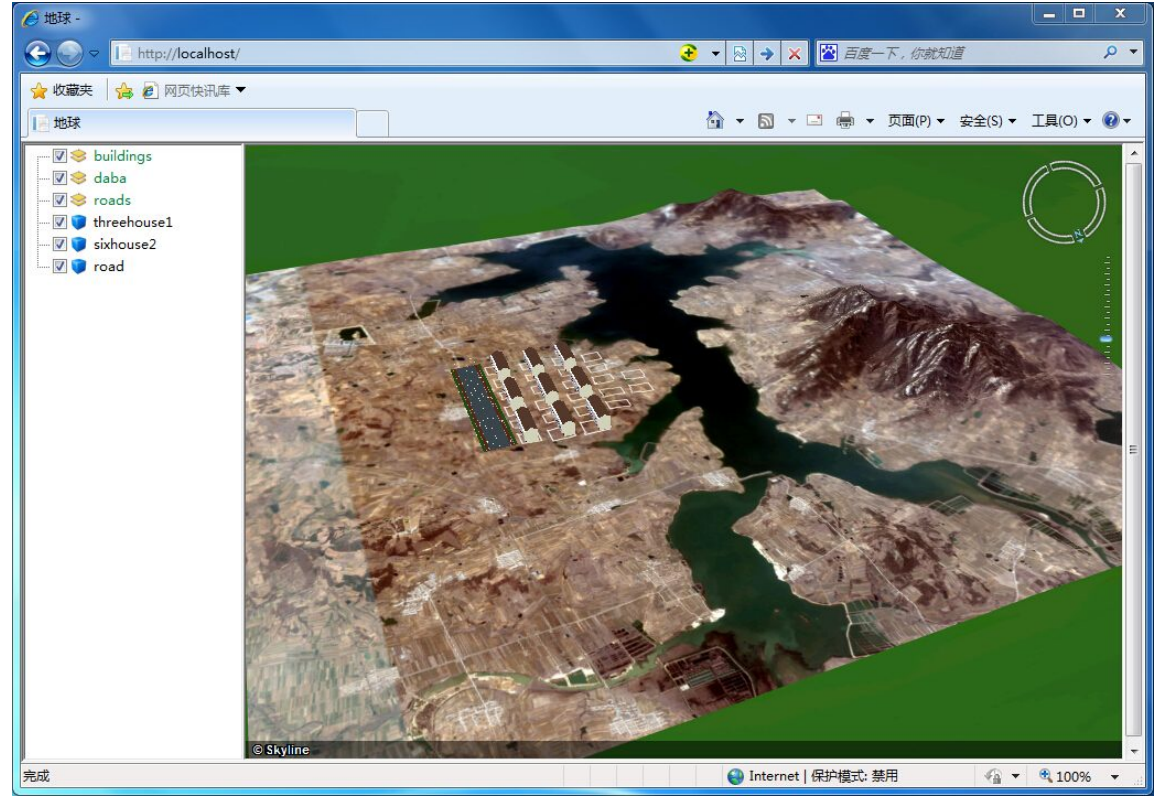

Figure 3. Viewing the scene on a page 


\subsection{Results discussion and description}

In this study, the three-dimensional terrain, basis of a three-dimensional scene, is built by the fusion of DEM data and real remote sensing images, of which the effect determines the fidelity of visual simulation. Landscape models can provide rendering for the scene thereby to improve its realism. SketchUp software can be used to create complex geometry models and add abundant texture, meanwhile the data volume of the models will greatly increase. The landscape models and scene in this study are relatively simple, while when the scene is more complex and with more models, the loading speed of the scene will be greatly reduced. Therefore, the structure and texture of model should be as simple as possible. The vector data provide positioning for landscape models in the paper and also can be used to achieve spatial analysis and attribute queries in a scene.

It still needs the support of TerraExplorer software for the client to browse three-dimensional terrain and scene directly published by TerraGate server and IIS server, thus lowering the use efficient of GIS data on the network. While the web browsing of the scene is achieved with common Internet Explorer browser thereby expanding the use of GIS data.

\section{CONCLUSIONS}

This paper combines network technology with 3D GIS technology and explores the establishment of network-based 3D GIS with Skyline software as platform. The main contents include construction of three-dimensional scene integrating three-dimensional terrain, landscape models and two-dimensional vectors, web publishing of terrain and scene data and web browsing of the scene. Utilizing technology of network, Web GIS and 3D visualization, this study achieves three-dimensional visual simulation of a watershed, so as to show its topographic features and provide a reference for related planning and construction of the watershed and flood control decision-making about the economic zone surrounding it. The following conclusions can be drawn through this study.

(1) This study publishes the scene data of the study area on the basis of Web GIS technology and the network architecture of $\mathrm{B} / \mathrm{S}$ mode, thus to offer browsing for remote users and achieve data sharing .

(2) The paper employs SketchUp software to create models for the major landscape within the study area, and integrates the models with other scene elements like three-dimensional terrain and two-dimensional vectors to accomplish visual simulation and reproduction of the terrain environment of the area.

\section{ACKNOWLEDGEMENTS}

The author are grateful for the support from Key Research Program of the Chinese Academy of Sciences( KZZD-EW-14), the National Science and Technology Support Program (2014FY210600), the National Natural Science Foundation of China (41171334), the Talent Fund of Yantai Institute of Coastal Zone Research, the Ecological Innovation \& Breeding Project (Y254021031, Y355031061) and the USDA NIFA Project (2010-34263-21075).

\section{REFERENCES}

[1] Chen, L., Xiang, N.P., Jiang, Y.P., and Zhong, J., "Research on Expression and Transmission of 3D Spatial Data Based on Web Services," Science of Surveying and Mapping, 35(3), 162-164 (2010).

[2] Chu, Z.W., and Yang, Y.L., "GIS Application Status and Development Trend," Modern Surveying and Mapping, 34(1), 19-22 (2011).

[3] Tang, Z.S., Wang, Y.G., Huang, J.F., Xin, Y., and Zhu, L.Y., "The Research of Internet 3DGIS Based on X3D," Bulletin of Surveying and Mapping, 32(9), 30-32 (2003).

[4] Hu, L.M., "Using Integrated Technology to Achieve Three-dimensional WebGIS System in Park Planning," Central South University, Hunan, China, Mater Thesis, (2011).

[5] Chang, J., "The Study on 3D Visualization of Flooding Scene Based on WebGIS," Zhengzhou University, Henan, China, Master Thesis, (2010).

[6] Zhang, C., "Application of WebGIS-based Three-dimensional Visualization Technology in Forest Fire Spread," Heilongjiang Science and Technology Information, (8), 82 (2008).

[7] Liang, J.X., and Zuo, X.Q., "Application and Research of Skyline in Web Three-dimensional GIS," Journal of Kunming University of Science and Technology ( Science and Technology), 34(2), 1-4 (2009). 
[8] Zhao, L.J., "System Development of Highway 3D GIS Based on Skyline," Proceedings of Computer Applications Branch of China Highway and Transportation Society, Hohhot, Inner Mongolia, 9-10 (2010).

[9] Hu, X.M., Sun, D.M., Huang, X.Y., and Hu, X.L., "Three Dimensional GIS System Design and Implementation Based on the Skyline: A Case Study of Chongqing Normal University," Modern City, (2), 15-18 (2012). 